\title{
O ensino de gerência em enfermagem na graduação: uma revisão integrativa
}

\section{The teaching of nursing management in undergraduate: an integrative review}

\author{
Mara Solange Gomes Dellaroza ${ }^{1}$; Cristiane Nakaya Tada ${ }^{2}$; Maria do Carmo \\ Lourenço Haddad $^{3}$; Marli Terezinha Oliveira Vannuchi'; Vanessa Gomes Maziero ${ }^{5}$
}

\section{Resumo}

O estudo tem como objetivo identificar a dinâmica do ensino de gerência na graduação de enfermagem. Trata-se de uma revisão integrativa de literatura com pesquisa realizada nas seguintes bases de dados on-line: Scielo, Medline, BDENF e Lilacs. As palavras chaves utilizadas no estudo foram: educação baseada em competências, competência profissional, educação em enfermagem, organização e administração, gerência, enfermagem, avaliação educacional, organização de serviços gerenciais, gerenciamento da prática profissional e ensino. Foram incluídos todos os artigos publicados nos últimos 10 anos que respondiam a questão norteadora que abordasse o ensino de gerência de enfermagem no Brasil, publicado em qualquer idioma. Foram excluídos os que não se apresentavam em texto completo. Dos 1432 estudos encontrados, após o cruzamento das palavras chaves, somente 8 foram selecionados por responderem ao objetivo desta revisão. A partir dos resultados encontrados, surgiram três categorias temáticas relacionadas: aos planos de ensino, carga horária e conteúdo programático; metodologias de ensino aprendizagem em gerência em enfermagem; desafios enfrentados no desenvolvimento do ensino de gerência em enfermagem.

Palavras-chave: Gerência. Educação em enfermagem. Administração em enfermagem. Ensino. Administração de serviços de saúde.

\section{Abstract}

The study aims to identify the dynamics of teaching management in nursing degree. It is an integrative literature review with research conducted in the following online database platforms: SciELO, Medline, Lilacs and BDENF. The keywords used in the study were: competency-based education, professional competence, education, nursing, organization and administration, management, nursing, educational assessment, organization of management services, management of professional practice and education. All articles published in the last 10 years which answered the question presented to approach the teaching of nursing management in Brazil, published in any language were included. Those that were not presented in full text were excluded. Of the 1432 studies identified after matching the keywords,

${ }^{1}$ Departamento de Enfermagem. Área de administração em enfermagem. Especialista em saúde do idoso e controle de dor.

${ }^{2}$ Graduação em Enfermagem pela Faculdade de Medicina de Marília.

${ }^{3}$ Doutorado em Enfermagem Fundamental pela Universidade de São Paulo. Professor Associado da Universidade Estadual de Londrina (UEL), Londrina-PR.

${ }^{4}$ Graduação em Farmácia Bioquímica e Enfermagem pela Universidade Estadual de Londrina. Mestrado em Ciências de Alimentos pela Universidade Estadual de Londrina. Doutorado em Saúde Pública pela Universidade de São Paulo. Professor Associado do curso de Graduação em Enfermagem da Universidade Estadual de Londrina (UEL), Londrina-PR.

${ }^{5}$ Graduação em Enfermagem pela Faculdade de Medicina de Botucatu - UNESP. Residência em Gerência de Serviços de Enfermagem pela Universidade Estadual de Londrina (UEL). Mestre pelo Programa de Pós-Graduação em Enfermagem Fundamental da Escola de Enfermagem de Ribeirão Preto (EERP) da Universidade de São Paulo - USP. 
only 8 were selected for answering objective of this review. From these results, three related themes emerged: the teaching plans, workload and program content; methodologies of teaching and learning in nursing management; challenges faced in developing the teaching of nursing management.

Keywords: Management. Nursing education. Teaching. Nursing administration. Health services administration.

\section{Introdução}

Com o mundo globalizado de hoje, caracterizado pela oferta de novas tecnologias de comunicação, de aparelhos eletrônicos e com inovações nos processos de trabalho e relações interprofissionais, torna-se indispensável a ocorrência de mudanças no ensino, considerando as diversas áreas (SILVA, 2009).

Assim, torna-se pertinente a presença de transformações no ensino na área da enfermagem, visto que o discente nos dias atuais, não é mais considerado espectador de seus conhecimentos, mas sujeito da construção deles, o qual advém da leitura, senso comum, história de vida, do contato humano e de produtos tecnológicos como a internet (SILVA, 2009).

A presença de mudanças e reformulações no ensino de gerenciamento de enfermagem torna-se necessária devido a importância da disciplina para a atuação do discente de enfermagem na prática profissional, que espera enfermeiros lideres no seu atuar.

A gerência, considerada uma ferramenta do processo do trabalho do cuidar, tem como foco de trabalho, os recursos humanos e a organização do trabalho, os quais têm como propósito a obtenção dos recursos humanos qualificados e um trabalho organizado, a fim de proporcionar uma assistência de enfermagem qualificada e integral a saúde (PERES, 2006). Constitui-se em atividade complexa que exige competências congnitivas, técnicas e atitudinais do enfermeiro, algo além do discurso teórico (JORGE, 2007).

O saber gerencial representa o fio condutor para o alcance do desenvolvimento profissional do enfermeiro (SILVA, 2000).
As diretrizes curriculares nacionais do curso de enfermagem, determina que o enfermeiro deve estar apto a realizar a administração e gerenciamento dos serviços de enfermagem considerada uma competência geral do enfermeiro, cabendo-lhe a ele gerenciar os recursos materiais, físicos e humanos, tornando-se um gestor e líder de equipes (BRASIL, 2000).

Para que o enfermeiro exerça a competência administrativa e gerencial, este deve possuir conhecimentos indispensáveis para a realização de um planejamento, para a tomada de decisão, interação e gerenciamento de recursos humanos (PERES, 2006).

Havendo o cumprimento das exigências propostas pelas novas Leis de Diretrizes e Base e das Diretrizes Curriculares Nacionais (DCN), a formação desses profissionais da saúde serão bem sucedidas a ponto de permitirem que os mesmos tenham uma boa atuação profissional com participação efetiva no sistema de saúde e com competência profissional, estando aptos para resolver os problemas de saúde da população (ITO, 2006).

Diante do exposto e considerando a importância do ensino de gerência em enfermagem para a formação o problema de pesquisa configurou-se na seguinte questão norteadora:

Como vem sendo desenvolvido o ensino de gerência em enfermagem durante a graduação no Brasil?

O objetivo do presente estudo foi identificar como vem sendo desenvolvido o ensino de gerência dos serviços de enfermagem na graduação. 


\section{Método}

Realizada uma revisão integrativa de literatura, pois este tipo de estudo permite que os profissionais de diversas áreas da saúde analisem os resultados relevantes de pesquisas, com a finalidade de ajudarem em suas tomadas de decisões e condutas, os quais proporcionam um saber crítico (MENDES, 2008).

Para tanto a pesquisa foi realizada obedecendo criteriosamente os seguintes passos: seleção da questão norteadora, estabelecimento dos critérios de inclusão e exclusão do estudo, representação das características do estudo original, análise dos dados, interpretação dos resultados e apresentação da revisão (FONSECA, 2009).

A coleta de dados foi realizada no mês de junho de 2011 contemplando as seguintes bases de dados on-line: Lilacs, Medline, Scielo e BDENF. Para a realização das buscas, foram utilizadas as seguintes palavras chaves presentes nos Descritores das Ciências da Saúde (DECS): educação baseada em competências, competência profissional, educação em enfermagem, organização e administração, gerência, enfermagem, avaliação educacional, organização de serviços gerenciais, gerenciamento da prática profissional e ensino.

Para a seleção dos artigos, foram incluídos todos os artigos publicados nos últimos 10 anos que respondiam a questão norteadora e que abordasse o ensino de gerência de enfermagem no Brasil, publicado em qualquer idioma.

Foram excluído todos os artigos que não estavam relacionados ao tema e que não foi possível obter o texto completo para análise.

Os estudos que obedeceram aos critérios de inclusão foram avaliados pelo pesquisador principal quanto à apresentação de informações relevantes para o objetivo desta revisão, por meio da análise do título e/ou resumo.

Os estudos que na leitura do resumo não constavam informações suficientes quanto ao objetivo do estudo, foram selecionados e posteriormente lidos na integra para a decisão de inclusão ou exclusão.

Posteriormente, foi construído um protocolo que possibilitou a análise clara e objetiva, com o auxílio de tabelas e quadros. Na etapa de análise dos dados buscou-se identificar os conteúdos abordados que respondam a questão norteadora e agrupa-las por temas formando assim as categorias apresentadas no resultado.

\section{Resultados}

Há necessidade de padronização: gerência de enfermagem? Administração de enfermagem?

Neste estudo, foram encontrados 1432 artigos após o cruzamento das palavras chaves. Com o objetivo de se obter a amostra do estudo, dos 1432 artigos encontrados, 1376 artigos foram excluídos com base na leitura dos títulos e 26 pela leitura dos resumos. A realização das exclusões dos artigos foi realizada com base nos critérios de inclusão e exclusão previamente estabelecidos. Os 30 artigos restantes, foram lidos na íntegra e destes, somente oito (8) foram selecionados para análise final do estudo.

Com relação aos periódicos nos quais os artigos selecionados foram publicados temos: Revista da Escola de Enfermagem da Universidade de São Paulo USP e a Revista Mineira de Enfermagem com publicação de um artigo, Revista Brasileira de Enfermagem e Revista Texto e Contexto e a Revista Latino Americana com publicações de dois artigos cada um.

Os anos de publicações dos artigos variaram entre 2001 a 2010, com destaque para o ano de 2006 com duas publicações. Nos anos de 2001, 2004, 2007, 2008, 2009 e 2010 foram publicados somente um artigo. 
Quadro 1 - Apresentação sobre o método dos estudos selecionados.

\begin{tabular}{|c|c|c|c|}
\hline Fonte & Periódico & Metodologia & Dados gerais sobre o Método \\
\hline $\begin{array}{l}\text { CIAMPONE } \\
2004\end{array}$ & enfer & $\begin{array}{r}\text { Quan } \\
\text { qualita }\end{array}$ & $\begin{array}{l}\text { Operacionalizada em duas etapas: a primeira teve como cenário, oito escolas e a segunda cinco, representativas } \\
\text { das regiões geopolíticas do país. Para a coleta de dados buscou-se os planos de ensino, considerando os } \\
\text { elementos: objetivos, carga horária, duração, inserção no curso, conteúdo, estratégias pedagógicas e forma } \\
\text { de avaliação. }\end{array}$ \\
\hline $\begin{array}{l}\text { KURCGANT } \\
2001\end{array}$ & $\begin{array}{l}\text { Rev. Esc. } \\
\text { Enferm. USP }\end{array}$ & $\begin{array}{l}\text { Qualitativa - } \\
\text { dialética }\end{array}$ & $\begin{array}{l}\text { O presente estudo está inserido em um projeto mais amplo, que vem sendo desenvolvido e que objetiva } \\
\text { conhecer como se dá a avaliação docente, discente e de resultados nas disciplinas que tratam de conteúdos } \\
\text { referentes à Administração em Enfermagem no âmbito dos cursos de Graduação e de Pós-Graduação no } \\
\text { Brasil. Neste estudo optou-se pelo reconhecimento do processo de avaliação a partir do referencial teórico } \\
\text { conforme diferentes abordagens pedagógicas, no sentido de desvendar as práticas do processo avaliativo, } \\
\text { buscando uma verdadeira práxis. }\end{array}$ \\
\hline $\begin{array}{l}\text { LOURENÇÃO } \\
2010\end{array}$ & enferm & Qualitativa & $\begin{array}{l}\text { Utilizou a análise de conteúdo dos documentos pedagógicos de um curso de enfermagem de uma instituição } \\
\text { de ensino superior da Região Sul do país. }\end{array}$ \\
\hline $\begin{array}{l}\text { NIMTZ } \\
2006\end{array}$ & $\begin{array}{l}\text { Rev Esc. } \\
\text { Enferm. USP }\end{array}$ & va & $\begin{array}{l}\text { Utilizado o método da análise de conteúdo em doze discursos de professores de Administração em } \\
\text { Enfermagem, em exercício nas Instituições Particulares de Ensino da Grande São Paulo, no período de } \\
\text { agosto a outubro de } 2002 \text {. }\end{array}$ \\
\hline $\begin{array}{l}\text { PERES } \\
2006 b\end{array}$ & $\begin{array}{r}\text { Texto cor } \\
\text { enfer }\end{array}$ & & $\begin{array}{l}\text { Realiza uma análise teórica das competências propostas nas Diretrizes Curriculares Nacionais da } \\
\text { Enfermagem }\end{array}$ \\
\hline $\begin{array}{l}\text { RESCK } \\
2008\end{array}$ & $\begin{array}{l}\text { Rev. } \\
\text { Latino-Am. } \\
\text { Enfermagem }\end{array}$ & $\begin{array}{l}\text { Qualitativa } \\
\text { dialética }\end{array}$ & $\begin{array}{l}\text { Envolveu } 11 \text { alunos, } 12 \text { docentes e } 12 \text { enfermeiros de serviço, partícipes do processo ensino-aprendizagem } \\
\text { do saber gerencial, no espaço intercessor, nos campos de prática e dos estágios curriculares, no ambiente } \\
\text { hospitalar e nos serviços básicos de saúde e do curso de graduação em enfermagem, de uma Instituição } \\
\text { Federal de Ensino Superior, da cidade de Alfenas, MG. Utilizou-se da técnica de coleta por grupo focal e da } \\
\text { análise de conteúdo. }\end{array}$ \\
\hline $\begin{array}{l}\text { ROTHBARTH } \\
2009\end{array}$ & $\begin{array}{l}\text { Texto contexto } \\
\text { enferm }\end{array}$ & Qualit & $\begin{array}{l}\text { Os sujeitos constituíram-se de sete enfermeiros que exercem docência em seis instituições de ensino superior } \\
\text { de Curitiba-PR. O período para a coleta de dados compreendeu os meses de abril a junho de 2008. A técnica } \\
\text { de coleta de dados foi a entrevista semi-estruturada, cujos dados foram submetidos à análise de conteúdo. }\end{array}$ \\
\hline $\begin{array}{c}\text { SANTOS } \\
2007\end{array}$ & $\begin{array}{l}\text { Rev. Min. } \\
\text { Enferm }\end{array}$ & $\begin{array}{l}\text { Quanti - } \\
\text { Qualitativa }\end{array}$ & $\begin{array}{l}\text { Para tanto, na primeira etapa, quantitativa, foi criado um instrumento com questões relativas aos quatro } \\
\text { pilares que compõem as competências para o gerenciamento: Saber fazer, Saber conhecer, Saber ser e Saber } \\
\text { conviver. Na segunda etapa, qualitativa, realizou-se um encontro de grupo focal para analisar a percepção do } \\
\text { graduando quanto ao desenvolvimento de suas competências }\end{array}$ \\
\hline
\end{tabular}

Fonte: Autor.

Para melhor compreensão do leitor, os resultados foram divididos em três categorias temáticas citados a seguir:

- Planos de ensino, carga horária e conteúdo programático;

- Metodologias de ensino aprendizagem em gerência em enfermagem;

- Desafios enfrentados no desenvolvimento do ensino de gerência em enfermagem;

\section{- Planos de ensino, carga horária e conteúdo programático:}

Quatro artigos abordaram a temática do plano de ensino. Em um estudo com o objetivo de identificar a metodologia de ensino e as bases pedagógicas utilizadas no ensino de administração em enfermagem, constatouse através de planos de aulas de 08 escolas, de graduação em enfermagem, que a carga horária utilizada para desenvolver a disciplina, teve variação de 150 horas até 1020 horas de carga horária. Das 08 escolas analisadas, somente uma escola, não constava o desenvolvimento desta disciplina na graduação (CIAMPONE, 2004).

Assim como no estudo citado anteriormente, uma pesquisa realizada com seis instituições de ensino superior em enfermagem da cidade de Curitiba consideradas do setor público e privado revela que o conteúdo de administração em enfermagem concentra em um único período, o oitavo período do curso. A carga horária variou de 306 a 792 horas incluindo teoria e prática (ROTHBARTH, 2009).

Quanto aos planos de ensino para o desenvolvimento da disciplina de administração em enfermagem, em um estudo, ressaltou-se que tem sido elaborado, na maioria das vezes, de forma tradicional, com objetivos amplos e fragmentados. Segundo dos autores: os conteúdos programáticos contidos nestes planos, não são problematizados de acordo com a atual política de saúde e de ensino do país, pois o enfoque se encontra de acordo com a abordagem tradicional da administração de enfermagem, com temas abarcados no modelo tecno- 
assistencial. Quanto aos planos de aulas específicos da disciplina de Administração, dos 52 títulos, 44 estavam associados à administração tradicional e 08 à Administração contemporânea. Dos 140 títulos de bibliografias contidos nos planos de aulas, 50 abarcavam assuntos relacionados a procedimentos, ou seja, abordavam as atividades do cuidar, embora tenham sido apresentados como conteúdos da administração de enfermagem (CIAMPONE, 2004).

De acordo com uma pesquisa, contida dentro da amostra do presente estudo, ressaltou-se que, a dimensão aprender a ser e a conviver não estão inseridas nos planos de ensino (NIMTZ, 2006).

Emum estudo com o objetivo de identificar a inserção das competências gerenciais por meio de documentos de uma instituição, como registros pedagógicos, mostrou-se que a habilidade de implementar e o desenvolvimento da dimensão atitude no processo de ensino aprendizagem pelo discente, é algo que merece atenção dentro do espaço pedagógico, com a finalidade de proporcionar ao aluno oportunidades do mesmo praticar o exercício da profissão durante a formação (LOURENÇÃO, 2010).

\section{- Metodologias de ensino aprendizagem em gerência em enfermagem}

Este tema foi o mais explorado nos artigos tendo sido abordado por sete artigos. As estratégias pedagógicas utilizadas no ensino de administração em enfermagem têm sido consideradas de caráter tradicional (KURCGANT, 2001), com predominância da abordagem da transmissão do conhecimento, havendo pouca valorização para as metodologias ativas (PERES, 2006a), os quais estimulam a participação efetiva do aluno no ensino (KURCGANT, 2001).

Segundo Ciampone e Kurcgant (2004) torna-se necessário a reformulação do processo de formação profissional na área de administração em enfermagem, principalmente nas práticas pedagógicas, com um ensino mais contextualizado propiciando a aprendizagem significativa com ênfase no aluno, estimulando-o a ser sujeito da construção de sua própria formação profissional (CIAMPONE, 2004).

O ensino descontextualizado, com a presença da dissociação entre teoria e a prática no ensino de administração em enfermagem, no decorrer da graduação de enfermagem foi considerado uma experiência não positiva para alunos de sétimo e oitavo semestre, pois no momento da vivência como enfermeiros, momento que exige o desenvolvimento de conhecimentos, habilidades e atitudes, ocorre a fragmentação teóricoprática. Desta forma, os autores analisam que o pensamento administrativo deveria ocorrer na medida em que vivenciam experiências nos estágio no decorrer da graduação (SANTOS, 2007).

Outro estudo reforça que as estratégias de ensino aprendizagem em administração em enfermagem devem ser pautadas por meio da problematização com a vivência do aluno em situações da prática profissional do enfermeiro (PERES, 2006a). Em um estudo com objetivo de identificar e caracterizar as práticas educativas desenvolvidas na disciplina de administração de enfermagem no âmbito de escolas de enfermagem do Brasil constatou-se que das 08 escolas estudadas, somente uma adota a problematização como metodologia de ensino. Ainda há a predominância da aula de cunho expositiva dialogada como abordagem teórica. Os estágios supervisionados, momento de desenvolvimento das habilidades e atitudes profissionais são trabalhados através de discussões em grupo e seminários (CIAMPONE, 2004).

Para Resck e Gomes (2008), a vivência do aluno em situações da prática do enfermeiro auxilia na consolidação das competências gerenciais para que se torne um processo contínuo, a ser construído no decorrer de sua vida profissional. Os autores afirmam que desenvolvimento das competências gerenciais se dá por meio da inter-relação constante entre a teoria e o estágio (RESCK, 2008). O ensino em administração em enfermagem, baseada em competências é considerado um potencial para proporcionar transformações positivas para este ensino, pois no ensino tradicional há a dissociação entre teoria e prática (PERES, 2006a). 
Quatro estudos abordam aspectos referentes aos docentes. Para os autores, as transformações devem ocorrer não somente nas práticas pedagógicas, mas também, nas competências docentes. $\mathrm{O}$ docente tem o papel de facilitar o aprendizado do aluno, de modo que este possa obter o conhecimento através de um olhar crítico da realidade em que está inserido. O pensamento crítico, e as novas tecnologias de ensino podem transformar o perfil do graduando com relação ao gerenciar. Para tanto, deve haver tanto o investimento nas tecnologias de ensino quanto um incentivo do docente como facilitador (KURCGANT, 2001). É indispensável o docente no auxílio da construção do conhecimento pelo aluno, tendo este o papel de discutir a relação teoria e prática das ações gerenciais do enfermeiro (ROTHBARTH, 2009).

Nesta linha, Nimtz e Ciampone (2006) reforçam ser necessário um gerenciamento eficaz do processo ensino aprendizagem pelo docente, com modificações nas práticas pedagógicas, e muito mais que isso, na mudança no comportamento destes, ou seja em suas atitudes (NIMTZ, 2006).

Esse preparo pedagógico pelo docente deve ocorrer principalmente com o surgimento de novas metodologias ativas, o qual proporciona ação-reflexão-ação, pois é necessário para que se proporcione um bom processo de ensino aprendizagem aos alunos (CIAMPONE, 2004).

\section{- Desafios enfrentados no desenvolvimento do ensino de gerência em enfermagem}

Sintetizamos a seguir a reflexão abordada pelos três artigos que abordaram esta temática.

Em um estudo com o objetivo de analisar a construção das competências gerenciais do acadêmico de enfermagem durante o estágio curricular, evidenciouse por meio de depoimentos de docentes e alunos que, o foco dos estágios na disciplina de administração em enfermagem tem-se voltado para a assistência, com poucas atenções para a gerência, as quais são desenvolvidas como atividades burocráticas, causando assim, uma imagem distorcida da ação gerencial (RESCK, 2008).
Nimtz e Ciampone (2006) refletem que a disciplina de administração de enfermagem, exige não somente dos alunos da graduação a utilização de suas habilidades de percepções a respeito da realidade em que estão, mas também dos professores fazendo com que tenham uma competência docente que abranja a percepção e sensibilidade para com o aluno, com descoberta dos seus anseios, desejos, e necessidades de aprendizado (ROTHBARTH, 2009).

Numa visão ampla do sistema de saúde trabalho aponta existir a divisão e a falta de parceria entre o serviço e o ensino, o que vai contra as políticas educacionais, que defendem a formação do futuro profissional no Sistema Único de Saúde (SUS) (PERES, 2006a).

\section{Discussão}

O intuito de investigar o modo de realização do ensino de gerência dos serviços de enfermagem veio a tona com a finalidade de se obter respostas e compreender o motivo dos enfermeiros, após a sua formação profissional, enfrentarem dificuldades em exercer a gerência dos serviços de enfermagem em seu local de trabalho. Questão considerada um problema atual, provocando, repercussões sistêmicas na assistência de enfermagem ao paciente, considerado o foco de trabalho. Através da análise deste estudo, foi possível elencar e analisar fatores que contribuem para o surgimento destas dificuldades que emergem durante o curso e se prolongam após a formação profissional.

De acordo com as pesquisas analisadas neste estudo, o ensino de gerência dos serviços de enfermagem vem enfrentando desafios, os quais compreendem desde a decisão da carga horária destinada à disciplina, ao estabelecimento do modo de construção dos planos de ensino na área, bem como a escolha da melhor metodologia de ensino a ser aplicada no ensino de gerência. 
Antes de propor soluções para os desafios enfrentados pelo ensino de gerência em serviços de enfermagem, é extremamente importante considerar as diretrizes curriculares nacionais, pois se trata de um grande norteador na área da educação.

Segundo as Diretrizes Curriculares Nacionais, a graduação tem como propósito formar enfermeiros crítico reflexivos, capazes de olhar para a realidade em que estão inseridos e intervirem sobre os problemas existentes, propondo soluções para a resolução destes. O profissional deve estar qualificado para exercer a profissão, exigindo assim, o senso de responsabilidade e cidadania, sempre compreendendo o paciente como um ser integral que possui seus aspectos biopsicosociais (BRASIL, 2000).

As diretrizes apontam que a falta de maturidade do aluno, e fatores intrínsecos ao aluno, como o interesse e motivação em desempenhar as competências gerenciais são também, considerados como desafios para o desenvolvimento de todas as competências gerenciais durante a graduação de enfermagem (BRASIL, 2000). Deve também, estar apto a ser um gestor de unidades de trabalho e líder de equipe com a responsabilidade de gerenciamento da unidade (SANTOS, 2007).

É a partir deste perfil profissional almejado, segundo a DCN, que as instituições de ensino superior em enfermagem devem organizar-se. Para tanto, há a necessidade que cada instituição de ensino tenha um olhar crítico para a realidade de ensino que possuem, a fim de construírem estratégias para estarem em concordância com as diretrizes vigentes.

Uma das estratégias, as quais podem ser utilizadas pelas instituições de ensino em enfermagem seria o investimento na elaboração dos planos de ensino pelos docentes, possibilitando a eles maior direcionamento no desenvolvimento da disciplina.
Segundo a análise dos estudos desta revisão, identificou-se que os planos de ensino elaborados por docentes da administração em enfermagem são caracterizados como tradicionais, com apresentação de objetivos amplos e fragmentados, segundo uma abordagem predominantemente tecno-assistencial quando deveria abordar temas específicos de gerência. Nesse aspecto, houve o desenvolvimento do tema com a abordagem tradicional, não vislumbrando as tendências contemporâneas, como a metodologia problematizadora.

Como solução para os desafios citado acima, os docentes da área especializadas deveriam valorizar a construção do planejamento didático antes do início de suas ações de ensino, com atenção específica aos objetivos a serem alcançados, aos recursos ou meios a serem utilizados, aos desafios a serem enfrentados, o tempo disponibilizado, bem como a avaliação final a ser realizada. Deve-se haver não só a valorização do planejamento didático do docente, mas também, o planejamento de sua prática, em que é indispensável à análise e reflexão sobre o perfil dos estudantes. $\mathrm{O}$ educador deve questionar para quem se destina o processo, quais suas necessidades e qual o perfil de profissional almeja formar de acordo com o planejamento construído (PEREIRA, 2007).

No momento da exposição didática é indispensável que haja a consideração do sujeito que vai a busca da construção de seu aprendizado e de quem ensina.

Outro desafio muito presente no ensino em gerência em enfermagem e que merece ser refletido está relacionado ao processo de ensino aprendizagem do discente.

De acordo com a análise deste estudo, identifica-se nos artigos a predominância da utilização de metodologias tradicionais no ensino de Administração de Enfermagem, baseada na transmissão do conhecimento. 
Neste tipo de metodologia, o professor tornase a principal fonte de informação e transmissor de uma verdade, podendo influenciar na atuação profissional futura, com limitações na capacidade de análise crítica e avaliativa dos problemas surgidos no local inserido e dificuldade no processo de busca de estratégias ou alternativas para a resolução do problema. O modelo tradicional de ensino aprendizagem não trabalha com o conflito entre os alunos, sendo desconsiderado o contexto em que estão inseridas, as diferentes visões e opiniões (CHIRELLI, 2004).

Desta forma, as instituições de ensino em enfermagem deveriam investir na utilização de metodologias ativas, como por exemplo: a problematização.

A problematização exige mudanças e transformações nas atitudes e postura do professor durante o ato de ensinar, pois educador tem como responsabilidade não somente estimular o exercício crítico-reflexivo do aluno, bem como, estar disponível para auxiliá-lo, de forma que possa colaborar com a construção de seu conhecimento. Um maior contato entre professor-aluno coloca o mesmo diante situações imprevistas e desconhecidas advindas da realidade social, exigindo, para tanto, que professor e aluno compartilhem, realmente, do processo de construção do aprendizado e não apenas da reconstrução e reelaboração dele (CYRINO, 2004).

A exigência de uma nova atuação com um desempenho diferenciado pelo professor, o qual tem como finalidade proporcionar o aprendizado significativo ao aluno, exige que o educador busque novos conhecimentos e habilidades no processo de ensino aprendizagem.

Para tanto, é indispensável, que as Escolas de Enfermagem do Brasil estruturem políticas de desenvolvimento de recursos humanos para o incentivo da capacitação docente, incluindo os da área de administração em enfermagem, com considerações às dimensões das competências, valores, atitudes, bem como a filosofia e estrutura organizacional do ensino (NIMTZ, 2006).

Este processo de capacitação docente deve ser um momento para proporcionar não somente a valorização da construção compartilhada dos saberes entre professores, mas do pensar conjunto para sanar as dificuldades e incertezas obtidas durante o processo de ensino, permitindo, ainda, acabar com as ações imediatistas, construindo assim, uma teoria emancipatória dando ao docente o direito de se tornar o sujeito de sua própria história profissional (NIMTZ, 2006).

\section{Conclusão}

A análise dos artigos dos últimos 10 anos sobre ensino de gerência de enfermagem demonstrou que esta disciplina ainda é muito pautada em metodologias tradicionais com o conteúdo baseado em linhas também tradicionais da antiga disciplina de administração. $O$ grande desafio apresentado nos resultados dos artigos é a mudança de paradigmas no ato de ensinar para uma pedagogia crítico reflexiva com abordagem de temas das atuais linhas de gerência contemporânea.

$\mathrm{O}$ ensino de gerência em enfermagem, disciplina desenvolvida no último período de graduação, é considerado essencial para a formação e desenvolvimento do estudante como futuro enfermeiro. Diante da relevância, acredita-se que os educadores da área devam realizar as reformulações de acordo com as tendências de ensino contemporâneo nas instituições de origem, visando a identificação dos problemas e de suas causas, seguindo para buscarem a resolutividade.

Nesta análise devem-se levar em consideração todos os fatores que influenciam direta e indiretamente no ensino em gerência em enfermagem, desde a simples elaboração dos planos de ensino até a atuação dos docentes e discentes, nunca perdendo de vista o real objetivo para a formação do enfermeiro. 


\section{Referências}

BRASIL. Ministério da Educação. Secretaria de Educação Superior. Departamento de Política do Ensino Superior. Comissão de Especialistas de Ensino de Enfermagem. Portaria $n^{\circ} 1518$, de 14 de junho de 2000. Dispõe sobre as diretrizes curriculares do curso de graduação em enfermagem. Brasília (DF): Ministério da Educação, 2000.

CHIRELLI, M. Q.; MISHIMA, S. M. O processo ensino aprendizagem crítico reflexivo. Revista Brasileira de Enfermagem, Brasília, v. 57, n. 3, p. 326-31, 2004.

CIAMPONE, M. H. T.; KURCGANT, P. O ensino de administração em enfermagem no Brasil: o processo de construção de competências gerenciais. Revista Brasileira de Enfermagem, Brasília, v. 57, n. 4, p. 401-7, 2004.

CYRINO, E. G.; TORALLES-PEREIRA, M. L. Trabalhando com estratégias de ensino-aprendizado por descoberta na área da saúde: a problematização e a aprendizagem baseada em problemas. Cadernos de Saúde Pública, Rio de Janeiro, v. 20, n. 3, p. 780788, 2004.

FONSECA, R. M. P.; PENICHE, A. C. G. Enfermagem em centro cirúrgico: trinta anos após a criação do Sistema de Assistência de Enfermagem Perioperatória. Acta Paulista de Enfermagem, São Paulo, v. 22, n. 4, p. 428-33, 2009.

ITO, E. E.; PERES, A. M.; TAKAHASHI, R. T.; LEITE, M. M. J. O ensino de enfermagem e as diretrizes curriculares nacionais: utopia $X$ realidade. Revista da Escola Enfermagem da USP, São Paulo, v. 40, n. 4, p. 570-5, 2006.

JORGE, M. S. B.; FREITAS, C. H. A.; NÓBREGA, M. F. B.; QUEIROZ, M. V. O. Gerenciamento em enfermagem: um olhar crítico sobre o conhecimento produzido em periódicos brasileiros (2000-2004). Revista Brasileira de Enfermagem, Brasília, v. 60, n. 1, p. 81-6, 2007.

KURCGANT, P.; CIAMPONE, M. H. T.; FELLI, V. E. A. Avaliação de desempenho docente, discente e de resultados na disciplina administração em enfermagem nas escolas de enfermagem no Brasil. Revista da Escola Enfermagem da USP, São Paulo, v. 35, n. 4 , p. 374-80, 2001.
LOURENÇÃO, D. C. A.; BENITO, G. A. V. Competências gerenciais na formação do enfermeiro. Revista Brasileira de Enfermagem, Brasília, v. 63, n. 1, p. 91-7, 2010.

MENDES, K. D. S.; SILVEIRA, R. C. C. P.; GALVÃO, C. M. Revisão integrativa: método de pesquisa para a incorporação de evidências na saúde e na enfermagem. Texto \& Contexto em Enfermagem, Florianópolis, v. 17, n. 4, p. 758-64, 2008.

NIMTZ, M. A.; CIAMPONE, M. H. T. O significado de competência para o docente de administração em enfermagem. Revista da Escola Enfermagem da USP, São Paulo, v. 40, n. 3, p. 336-42, 2006.

PEREIRA, S. E. Contribuições para um planejamento educacional em ciências da saúde com estratégias inovadoras de ensino-aprendizagem. Comunicação em Ciências da Saúde, Brasília, v. 18, n. 1, p. 33-44, 2007.

PERES, A. M. Competências gerenciais do enfermeiro: relação entre as expectativas da instituição formadora e do mercado de trabalho. 2006. 250 f. Tese (Doutorado em Enfermagem) - Escola de Enfermagem da Universidade de São Paulo, São Paulo, 2006.

PERES, A. M.; CIAMPONE, M. H. T. Gerência e competências gerais do enfermeiro. Texto \& Contexto em Enfermagem, Florianópolis, v. 15, n. 3, p. 492-9, 2006.

RESCK, Z. M. R.; GOMES, E. L. R. A formação e a prática gerencial do enfermeiro: caminhos para a práxis transformadora. Revista Latino-Americana de Enfermagem, Ribeirão Preto, v. 16, n. 1, p. 71-7, 2008.

ROTHBARTH, S.; WOLFF, L. D. G; PERES, A. M. O desenvolvimento de competências gerenciais do enfermeiro na perspectiva de docentes de disciplinas de administração aplicada à enfermagem. Texto \& Contexto em Enfermagem, Florianópolis, v. 18, n. 2, p. 321-9, 2009.

SANTOS, E. P.; CIAMPONE, M. H. T. Avaliação de competências gerenciais: a percepção de alunos do curso de graduação em enfermagem da USP. Revista Mineira de Enfermagem, Belo Horizonte, v. 11, n. 4, p. 395-401, 2007. 
SILVA, R. M.; SILVA, I. C. M.; RAVALIA, R. A. Ensino de enfermagem: reflexões sobre o estágio curricular supervisionado. Revista Práxis, Volta Redonda, v. 1, n. 1, p. 37-41, 2009.

SILVA, V. R. A prática gerencial do enfermeiro em instituições hospitalares. 2000. 277 f. Dissertação (Mestrado em Enfermagem) - Escola Paulista de Medicina/UNIFESP, São Paulo, 2000. 